\section{Cureus}

Received 05/29/2018

Review began 06/02/2018

Review ended 07/09/2018

Published 07/23/2018

\section{(c) Copyright 2018}

Bahl et al. This is an open access article distributed under the terms of the Creative Commons Attribution License CC-BY 3.0., which permits unrestricted use, distribution, and reproduction in any medium, provided the original author and source are credited.

\title{
'Bleedling Dilemma': The Story of a Periampullary Mass
}

Bhavyaa Bahl ${ }^{1}$, Rohith Vadlamudi ${ }^{2}$, Parekha Yedla ${ }^{3}$, Roger D. Smalligan ${ }^{4}$

1. UAB Internal Medicine, Huntsville Regional Medical Campus, Huntsville Hospital, Huntsville, USA 2. UAB School of Medicine, Huntsville Regional Medical Campus, Huntsville Hospital, Huntsville, USA 3. UAB Internal Medicine, Huntsville Regional Medical Campus, Huntsville Hospital, Huntsville, USA 4. UAB Medicine, Huntsville Regional Medical Campus, Huntsville Hospital, Huntsville, USA

$\square$ Corresponding author: Bhavyaa Bahl, bhavyaabahl@uabmc.edu Disclosures can be found in Additional Information at the end of the article

\section{Abstract}

Periampullary malignancies arise in the vicinity of the ampulla of Vater, a common passage for biliary and pancreatic secretions. Determining the anatomical origin of these tumors represents a diagnostic challenge. This is especially true for large tumors due to the transitional nature of this region, proximity to different structures, anatomical variations, and overlapping features among constituting structures. This determination has significant prognostic and therapeutic implications. Among them, primary ampullary adenocarcinoma is a rare malignancy that has the best overall prognosis with high rates of potentially curative resection and possible survival even in advanced disease. Due to its rarity, it is also a vague territory with no definitive guidelines regarding management and surveillance currently available. Acute gastrointestinal hemorrhage is a rare presentation of ampullary carcinoma that occurs secondary to tumor ulceration.

We report an elderly male with a previously known large, initially asymptomatic periampullary mass who came for evaluation of melena and was noted to be hypotensive secondary to acute blood loss from the large tumor, later determined to be adenocarcinoma of the ampulla of Vater.

Categories: Internal Medicine, Gastroenterology, Oncology

Keywords: cancer of ampulla of vater, periampullary mass, coil embolization, ampullary adenocarcinoma, gastroduodenal artery, gastrointestinal bleeding, angiography, melena, pancreatoduodenectomy, ampullectomy

\section{Introduction}

The ampulla of Vater is an anatomically complex structure within the major duodenal papilla. It is a dilated passage at the confluence of the common bile duct and the main pancreatic duct, which drains their respective secretions into the second part of the duodenum through the papillary opening [1]. There are many anatomical variants of this arrangement. The sphincter of Oddi is a muscular valve consisting of smooth muscles surrounding the distal part of the common bile duct (CBD), the main pancreatic duct, and the ampulla. Tumors arising in this region are collectively referred to as periampullary and they may originate from any of the structures in the vicinity of the ampulla including pancreas, CBD, duodenum and the ampulla itself.

Despite being the most common site of neoplastic transformation in the small intestine, 
primary ampullary malignancies are rare with an incidence of four to six per million representing $0.5 \%$ of all gastrointestinal and $7 \%$ of all periampullary malignancies [1-3]. Some $90 \%$ of these are ampullary adenocarcinomas, which usually present as jaundice, pruritis, abdominal pain, nausea, dyspepsia, weight loss, melena, malabsorptive diarrhea, and fatigue. The strategic location of the tumor in the bile outflow tract leads to obstructive jaundice in up to $72 \%-90 \%$ cases allowing for the detection of small tumors early in the course of malignancy [4].

Both benign and malignant ampullary tumors can occur sporadically or in association with a genetic syndrome. The incidence of these tumors has shown dramatic increment in association with hereditary polyposis syndromes like familial adenosis polyposis (FAP) and hereditary nonpolyposis colorectal cancer (HNPCC) [1]. These may be diagnosed earlier than the sixth or seventh decade of life, the average age of diagnosis for sporadic cases [1-2].

We present an elderly male with multiple comorbidities brought for evaluation of melena, a year after a prior incidence of hematemesis. Evaluation of hematemesis on prior admission had revealed a large periampullary mass that remained untreated. It was determined to be the cause of acute gastrointestinal hemorrhage leading to hemodynamic instability, a rare association with ampullary adenocarcinoma.

\section{Case Presentation}

A 79-year-old African American male was admitted for evaluation of two episodes of melena within one day. No associated abdominal pain, nausea, weight loss, appetite changes, diarrhea, hematemesis, or hematochezia was reported. His past medical history was significant for chronic obstructive pulmonary disease (COPD), heart failure with reduced ejection fraction of $25 \%$, coronary artery disease, dementia, and a recent large left middle cerebral artery (MCA) stroke that had led to aphasia and residual right hemiparesis.

The patient was admitted a year ago for evaluation of hematemesis with a hemoglobin level of $6.9 \mathrm{~g} / \mathrm{dL}$. At that time, esophagogastroduodenoscopy (EGD) had shown a large submucosal, ulcerated mass in the area of major duodenal papilla with histology suggestive of benign small intestinal mucosa without any atypical changes (Figure 1). A subsequent computed tomography (CT) scan of abdomen and pelvis confirmed a $6.7 \mathrm{~cm}$ x $5.5 \mathrm{~cm}$ mass at the pancreatic head invading the duodenum. It had led to a pancreatic duct dilatation of $11 \mathrm{~mm}$ seen as a cut-off sign on CT. Endoscopic ultrasound (EUS) to characterize the mass had to be terminated prematurely due to hypotension at the beginning of the procedure. He was eventually discharged after stabilization of his vitals and hemoglobin for a repeat outpatient EUS within a week. He failed to follow up with his appointment. 


\section{Cureus}

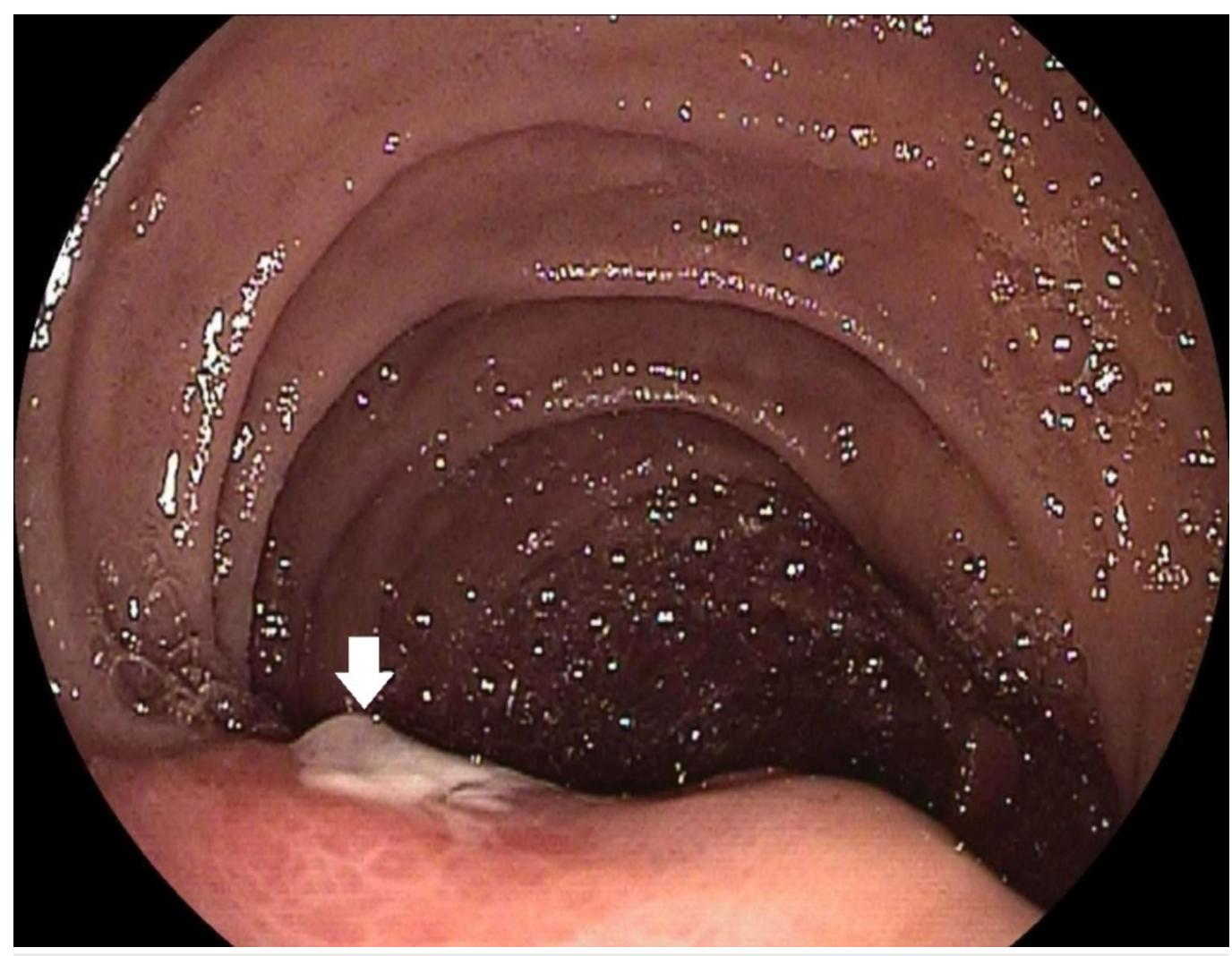

\section{FIGURE 1: Esophagogastroduodenoscopy (EGD) on prior}

admission.

Front-viewing endoscope showing an ulcerated submucosal mass at the duodenal papilla with no apparent bleeding.

Examination on this admission revealed an ill-appearing, aphasic, thin male with hypotension and tachycardia. Initial testing showed a hemoglobin level of $9.9 \mathrm{~g} / \mathrm{dL}$, a blood urea nitrogen (BUN) level of 30, an international normalized ratio (INR) of 1.1, and a total bilirubin level of 0.3. After initial resuscitation with intravenous fluids and red blood cell transfusions, an emergent EGD was performed using front- and side-viewing endoscope. A fungating, polypoid mass was seen within the ampulla of Vater with blood oozing out of the duodenal papilla that failed to be controlled with epinephrine injection (Figure 2). A hypervascular mass was seen within the second part of duodenum and pancreatic head with active hemorrhage from the supplying vessels including the superior pancreaticoduodenal branch of gastroduodenal artery (GDA) on the following arteriogram. Successful coil embolization of GDA was able to control bleeding and the patient did not require any further transfusions. 


\section{Cureus}

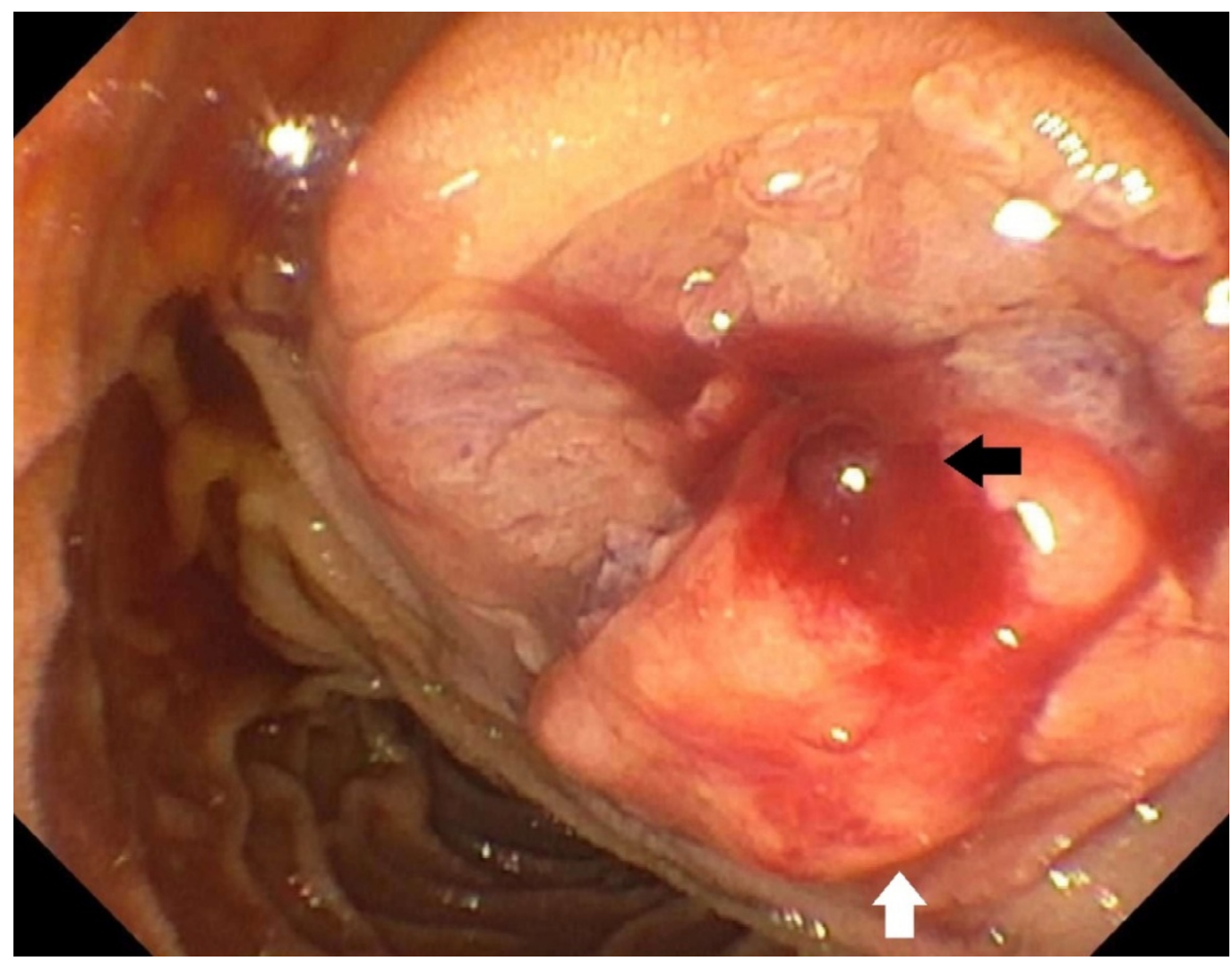

FIGURE 2: EGD on reported admission.

Side-viewing endoscope showing a fungating, polypoid mass (white arrow) with blood oozing from the duodenal papilla (black arrow).

A biopsy specimen taken during endoscopy showed 'invasive adenocarcinoma' on histopathology (Figure 3). CA 19-9 level was within the reference range $15.6 \mathrm{U} / \mathrm{mL}(0-37 \mathrm{U} / \mathrm{mL})$. Based on the clinical picture, imaging and pathology data, he was diagnosed with adenocarcinoma of the ampulla of Vater.

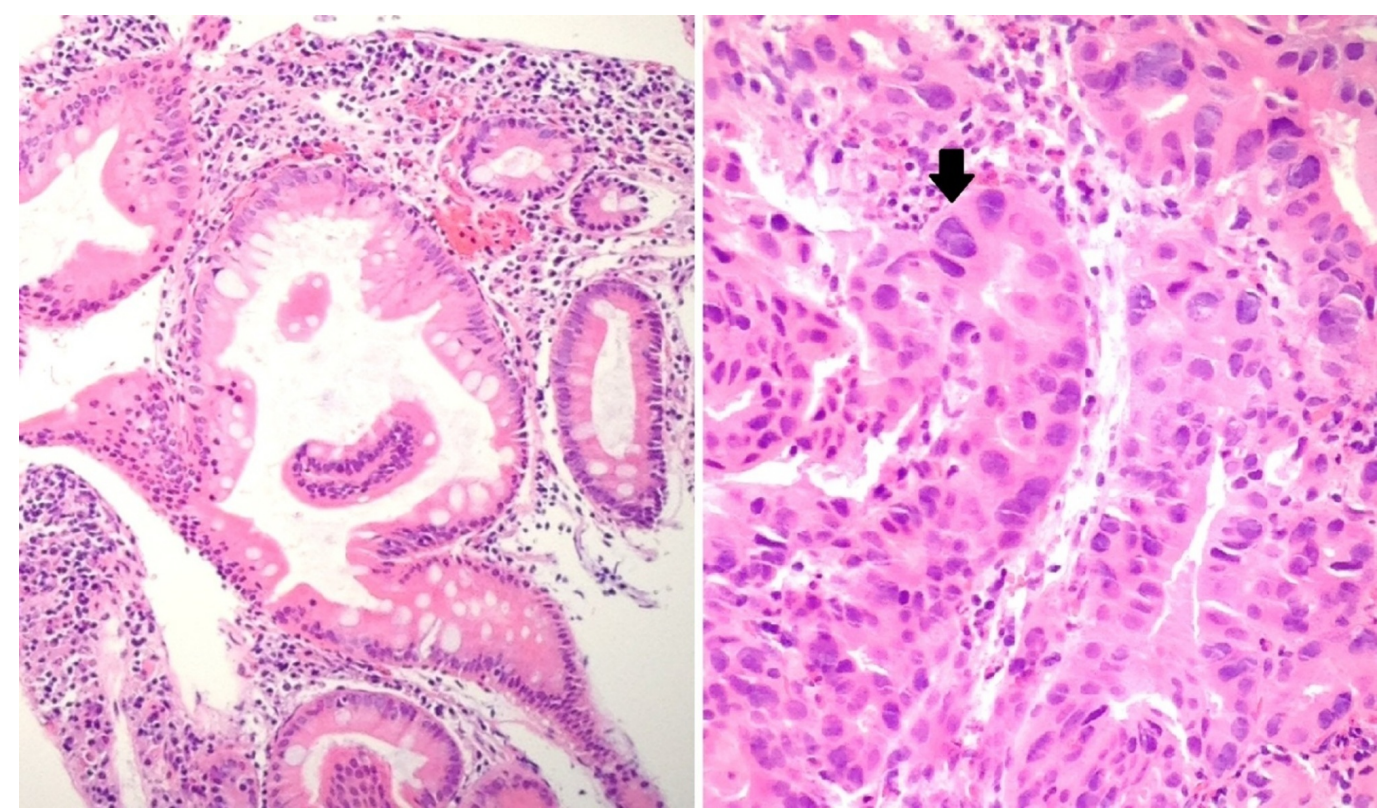


FIGURE 3: Histology of biopsy specimen from the ampulla of Vater using H\&E, 100x.

Left : Normal ampullary mucosa with intestinal differentiation.

Right : Invasive ampullary adenocarcinoma with cellular atypia (black arrow).

Due to his poor performance status and multiple comorbidities, he was not considered as a candidate for surgery or aggressive chemotherapy. He refused any aggressive measures and was discharged to a nursing facility with an outpatient oncology follow up for consideration of palliative radiotherapy.

\section{Discussion}

Malignancies in the periampullary region are diagnosed based on the endoscopic, radiologic, and histopathologic data.

Ultrasound (US), CT, magnetic resonance imaging (MRI), magnetic resonance cholangiopancreatography (MRCP), or endoscopic retrograde cholangiopancreatography (ERCP) is used for evaluation of this region. The EUS is used only in a few cases requiring further delineation where there is suspicion for malignancy despite negative biopsy similar to the case described. It gives the most accurate assessment of the $\mathrm{T}$ stage along with tumor extension and penetration within the duct and surrounding tissues, respectively. The recent use of intraductal ultrasound (IDUS) to visualize and explore bile and pancreatic ducts has increased the diagnostic accuracy [5].

Large periampullary malignancies like the one presented, represent a diagnostic challenge in terms of determining the anatomical origin [6]. The importance of this determination has prognostic and therapeutic implications, which differ based on the tissue of origin. In such cases where the usual diagnostic modalities fail, a diagnosis may be reached once gross dissection and histopathology evaluation is performed after a complete surgical resection. Gross dissection to understand the relationship between the structures of these complex forms the basis of such a determination [6]. The clue to a particular origin may lie in the presence of a corresponding preinvasive disease on histological evaluation [6].

In this case, the tumor was considered to be ampullary and not pancreatic in origin due to a more indolent course of progression. Pancreatic adenocarcinomas have a highly aggressive course with a poor prognosis and a five-year survival rate of only three percent in contrast to 30\%-50\% noted in adenocarcinomas of ampullary origin with limited nodal involvement [1]. This malignancy was $\mathrm{T} 3$ tumor stage at the time of detection and did not show any progression a year later even in the absence of therapy.

Further histologic subdivision of ampullary adenocarcinoma into intestinal and pancreatobiliary subtype was first demonstrated by Kimura et al. in 1994 [7]. Histomolecular staining is now used to differentiate the two subtypes. This has been considered important due to a worse prognosis associated with the pancreaticobiliary subtype first demonstrated in a study by Westgaard et al. in 2008 although the evidence at this point is unclear [8-9]. The intestinal subtype stains positive for $\mathrm{CK} 20$, MUC-2, and CDX-2 whereas the pancreaticobiliary subtype stains for CK 17, MUC-1 and MUC5AC, also shared by pancreatic adenocarcinoma (PDAC) [6]. In about five percent cases, a mixed pattern consisting of both intestinal and pancreaticobiliary 
glands is noted [6].

The 2017 American Joint Committee on Cancer (AJCC)/Union for International Cancer Control (UICC) TNM system is used for staging of ampullary adenocarcinoma. In nonmetastatic disease, prognosis mainly depends upon the extent of tumor invasion and lymphatic spread represented by the $\mathrm{T}$ and $\mathrm{N}$ stage, respectively.

Despite pancreatic duct obstruction secondary to a T3 stage tumor, our patient did not report any typical symptoms. These tumors are a rare cause of obscure gastrointestinal bleeding and more rarely, can also lead to overt hemorrhage secondary to tumor ulceration. This is seen as blood oozing out of the major duodenal papilla on a side-viewing endoscope which also facilitates visualization and biopsy of the ampullary mass. This may initially mimic other rare causes of obscure gastrointestinal bleeding (OGIB) including hemobilia and hemosuccus pancreaticus, which refer to bleeding originating in the CBD and pancreatic duct, respectively. The differentiation may become apparent during angiography when the source and tract of bleeding are delineated. The intermittent nature of bleeding in these conditions contributes to their obscurity. The CT angiogram (CTA), a noninvasive procedure has a sensitivity and specificity of 79\%-90\% and 95\%-99\% [10-11], respectively, in the detection of OGIB with a diagnostic accuracy of $100 \%$ for bleeding at a rate of $0.3-0.5 \mathrm{~mL} / \mathrm{min}$ [12]. It is among the firstline diagnostic procedures that should be considered in a hemodynamically unstable obscure gastrointestinal bleed. Its major limitation is a lack of therapeutic application.

On the other hand, invasive angiography has both diagnostic and therapeutic applications. It can detect bleeding $>0.5 \mathrm{~mL} / \mathrm{min}$ with a sensitivity and specificity of $30 \%-47 \%$ and near $100 \%$, respectively [13-14]. It may be used as an adjunct for therapeutic embolization after CTA in a hemodynamically unstable patient with brisk bleeding or as the only angiography procedure performed for both diagnostic and therapeutic purposes if the bleeding source is known and there has been a failure to achieve hemostasis despite the use of pharmacologic/endoscopic methods as described in the case discussed. Angiographic methods to control bleeding include injecting vasoactive agents like vasopressin or using agents to mechanically occlude the bleeding vessel, a process called embolization. Commonly used embolic agents are gelatin sponges, polyvinyl alcohol (PVA) particles, acrylic microspheres, and steel coils. Surgery is indicated in a few cases where the source is known, an increasing need for transfusions is noted, or there is life-threatening bleeding from a defined origin [12].

It is important to recognize the limitation of using a front-viewing endoscope while looking for the source of an OGIB as it can easily miss lesions of the ampullary complex due to its location. Endoscopic sampling is associated with a high false-negative histopathology nearing 50\% due to sampling difficulties that could yield inadequate specimen. Side-viewing ERCP endoscope is able to identify causes in the gut wall and must be considered while evaluating the duodenal papilla and the ampullary complex.

Pancreatoduodenectomy (PD) or Whipple's procedure is the standard surgical procedure for ampullary cancer with potentially curative resection achieved in as high as $90 \%$ cases [15]. Fiveyear survival rates after PD with and without lymph node involvement are 17\%-50\% and 64\%$80 \%$, respectively [16]. Postoperative complications including pancreatic fistula, anastomotic leaks, delayed gastric emptying, intra-abdominal infections, and pneumonia are seen in up to $20 \%-40 \%$ cases [16]. The high postoperative morbidity limits the number of patients considered suitable to undergo PD.

Ampullectomy is an option that can be considered in early, limited lesions $<1 \mathrm{~cm}$ when the patient is unsuitable to undergo PD, although it is not an alternative to it. An aggressive preoperative and intraoperative assessment is needed to rule out necessary criteria like tumor 
invasion, high-grade morphology, lymphovascular invasion, and lymph node involvement for such a consideration. The risk of tumor recurrence and shorter disease-free survival in comparison to PD is the most important limiting factor [17].

Ampullectomy can be performed as a minimally invasive transduodenal surgery or as an endoscopic procedure. Endoscopic ampullectomy is the surgery of choice for benign ampullary lesions $<20 \mathrm{~mm}$ including lesions with low-grade dysplasia [18]. It may be considered in highgrade dysplasia and carcinoma in situ (Tis) after an appropriate staging and assessment. Lack of lymphadenectomy and proper pathological staging using this approach further increase the chances of tumor recurrence by many folds.

Transduodenal surgical ampullectomy is an infrequently performed procedure which is less invasive than PD and has a lower surgical morbidity. It can be considered in Tis and T1 disease with lesions $<1 \mathrm{~cm}$ [17]. There are no uniform inclusion criteria for local resection. As a rule, PD should be performed whenever possible.

There is a lack of specific guidelines regarding the management and surveillance of this malignancy. Despite good resectability, recurrence remains a major issue. There is no consensus regarding the use of adjuvant chemotherapy due to the rarity of the condition, lack of definitive data, and uncertainty of benefits. However, most physicians offer adjuvant chemotherapy with (USA) or without (Europe) chemoradiation due to high recurrence rates. The drug regimen is on the lines of adjuvant chemotherapy for resected pancreatic adenocarcinoma based on fluoropyrimidine or gemcitabine [19-20]. Chemotherapy for metastatic disease based on the results of phase III ABC-02 trial consists of gemcitabine and cisplatin [19].

Poor prognostic factors include the presence of nodal metastasis, poor differentiation on histology, tumor invasion, and positive postoperative surgical margins. Other factors associated with an adverse outcome are macroscopic ulceration and perineural, lymphatic, or vascular invasion [15].

Despite a large number of studies regarding the management and prognosis of ampullary adenocarcinoma, there remains significant ambiguity evident by lack of treatment and surveillance guidelines. Our understanding remains limited due to the small sample size of these studies, rarity of the disease, and the dilemma posed by periampullary tumors. Hopefully, the increasing incidence as reported in a few studies will be met with defined guidelines in the near future [3].

\section{Conclusions}

Tumors of the periampullary region represent a diagnostic challenge. Determining the tissue of origin in such cases is necessary for appropriate management and prognosis. Among these, ampullary cancer represents a rare category that has a more favorable prognosis than others. Acute gastrointestinal hemorrhage secondary to such a lesion is a rare presentation which may mimic conditions like hemobilia or hemosuccus pancreaticus requiring emergent management. Due to its rarity and lack of large multi-center trials, currently, there are no definitive guidelines for the management and surveillance of ampullary cancer. This has presented as an area of ambiguity for a disease considered to have a much better prognosis than all other periampullary tumors.

\section{Additional Information \\ Disclosures}


Human subjects: Consent was obtained by all participants in this study. Conflicts of interest: In compliance with the ICMJE uniform disclosure form, all authors declare the following:

Payment/services info: All authors have declared that no financial support was received from any organization for the submitted work. Financial relationships: All authors have declared that they have no financial relationships at present or within the previous three years with any organizations that might have an interest in the submitted work. Other relationships: All authors have declared that there are no other relationships or activities that could appear to have influenced the submitted work.

\section{References}

1. Panzeri F, Crippa S, Castelli P, et al.: Management of ampullary neoplasms: a tailored approach between endoscopy and surgery. World J Gastroenterol. 2015, 21:7970-7987. 10.3748/wjg.v21.i26.7970

2. Rostain F, Hamza S, Drouillard A, et al.: Trends in incidence and management of cancer of the ampulla of Vater. World J Gastroenterol. 2014, 20:10144-10150.

10.3748/wjg.v20.i29.10144

3. Albores-Saavedra J, Schwartz AM, Batich K, et al.: Cancers of the ampulla of vater: demographics, morphology, and survival based on 5,625 cases from the SEER program. J Surg Oncol. 2009, 100:598-605. 10.1002/jso.21374

4. Tsukada K, Takada T, Miyazaki M, et al.: Diagnosis of biliary tract and ampullary carcinomas . J Hepatobiliary Pancreat Surg. 2008, 15:31-40. 10.1007/s00534-007-1278-6

5. Castillo C: Endoscopic ultrasound in the papilla and the periampullary region. World J Gastrointest Endosc. 2010, 2:278-287. 10.4253/wjge.v2.i8.278

6. Bledsoe JR, Shinagare SA, Deshpande V: Difficult diagnostic problems in pancreatobiliary neoplasia. Arch Pathol Lab Med. 2015, 139:848-857. 10.5858/arpa.2014-0205-RA

7. Kimura W, Futakawa N, Yamagata S, et al.: Different clinicopathologic findings in two histologic types of carcinoma of papilla of Vater. Jpn J Cancer Res. 1994, 85:161-166. 10.1111/j.1349-7006.1994.tb02077.x

8. Westgaard A, Tafjord S, Farstad IN, et al.: Pancreatobiliary versus intestinal histologic type of differentiation is an independent prognostic factor in resected periampullary adenocarcinoma. BMC Cancer. 2008, 8:170. 10.1186/1471-2407-8-170

9. Zhou H, Schaefer N, Wolff M, et al.: Carcinoma of the ampulla of Vater: comparative histologic/immunohistochemical classification and follow-up. Am J Surg Pathol. 2004, 28:875-882. 10.1097/00000478-200407000-00005

10. Kennedy DW, Laing CJ, Tseng LH, et al.: Detection of active gastrointestinal hemorrhage with CT angiography: a 4(1/2)-year retrospective review. J Vasc Interv Radiol. 2010, 21:848-855. 10.1016/j.jvir.2010.01.039

11. Yoon W, Jeong YY, Shin SS, et al.: Acute massive gastrointestinal bleeding: detection and localization with arterial phase multidetector row helical CT. Radiology. 2006, 239:160-167. 10.1148/radiol.2383050175

12. Sánchez-Capilla AD, De La Torre-Rubio P, Redondo-Cerezo E: New insights to occult gastrointestinal bleeding: from pathophysiology to therapeutics. World J Gastrointest Pathophysiol. 2014:271-283. 10.4291/wjgp.v5.i3.271

13. Graça BM, Freire PA, Brito JB, et al.: Gastroenterologic and radiologic approach to obscure gastrointestinal bleeding: how, why, and when?. Radiographics. 2010, 30:235-252. 10.1148/rg.301095091

14. Fiorito JJ, Brandt LJ, Kozicky O, et al.: The diagnostic yield of superior mesenteric angiography: correlation with the pattern of gastrointestinal bleeding. Am J Gastroenterol. 1989, 84:878-881.

15. Lazaryan A, Kalmadi S, Almhanna K, et al.: Predictors of clinical outcomes of resected ampullary adenocarcinoma: a single-institution experience. Eur J Surg Oncol. 2011, 37:791797. 10.1016/j.ejso.2011.06.008

16. Allema JH, Reinders ME, van Gulik TM, et al.: Results of pancreaticoduodenectomy for ampullary carcinoma and analysis of prognostic factors for survival. Surgery. 1995, 117:247253. 10.1016/S0039-6060(05)80197-7

17. Yoon Y-S, Kim S-W, Park SJ, et al.: Clinicopathologic analysis of early ampullary cancers with 


\section{Cureus}

a focus on the feasibility of ampullectomy. Ann Surg. 2005, 242:92-100.

10.1097/01.sla.0000167853.04171.bb

18. Bassan M, Bourke M: Endoscopic ampullectomy: a practical guide. J Interven Gastroenterol. 2012, 2:23-30. 10.4161/jig.20131

19. Valle J, Wasan H, Palmer DH, et al.: ABC-02 Trial Investigators. Cisplatin plus gemcitabine versus gemcitabine for biliary tract cancer. N Engl J Med. 2010, 362:1273-1281.

10.1056/NEJMoa0908721

20. Neoptolemos JP, Moore MJ, Cox TF, et al.: Effect of adjuvant chemotherapy with fluorouracil plus folinic acid or gemcitabine vs observation on survival in patients with resected periampullary adenocarcinoma: the ESPAC-3 periampullary cancer randomized trial. J Am Med Assoc. 2012, 308:147-156. 10.1001/jama.2012.7352 